\title{
A BLOW-UP CRITERION FOR A DEGENERATE PARABOLIC PROBLEM DUE TO A CONCENTRATED NONLINEAR SOURCE
}

\author{
BY \\ C. Y. CHAN (Department of Mathematics, University of Louisiana at Lafayette, Lafayette, \\ Louisiana 70504-1010)
}

AND

R. BOONKLURB (Department of Mathematics, University of Louisiana at Lafayette, Lafayette, Louisiana 70504-1010)

Abstract. Let $q, a, b$, and $T$ be real numbers with $q \geq 0, a>0,0<b<1$, and $T>0$. This article studies the following degenerate semilinear parabolic first initial-boundary value problem,

$$
\begin{gathered}
x^{q} u_{t}(x, t)-u_{x x}(x, t)=a \delta(x-b) f(u(x, t)) \text { for } 0<x<1,0<t \leq T, \\
u(x, 0)=\psi(x) \text { for } 0 \leq x \leq 1, u(0, t)=u(1, t)=0 \text { for } 0<t \leq T,
\end{gathered}
$$

where $\delta(x)$ is the Dirac delta function, and $f$ and $\psi$ are given functions. It is shown that for $a$ sufficiently large, there exists a unique number $b^{*} \in(0,1 / 2)$ such that $u$ never blows up for $b \in\left(0, b^{*}\right] \cup\left[1-b^{*}, 1\right)$, and $u$ always blows up in a finite time for $b \in\left(b^{*}, 1-b^{*}\right)$. To illustrate our main results, two examples are given.

1. Introduction. Let $q, a, b$, and $T$ be real numbers with $q \geq 0, a>0,0<b<1$, and $T>0, L u=x^{q} u_{t}-u_{x x}, D=(0,1), \bar{D}=[0,1]$, and $\Omega=D \times(0, T]$. We consider the following degenerate semilinear parabolic first initial-boundary value problem,

$$
\left.\begin{array}{c}
L u=a \delta(x-b) f(u(x, t)) \text { in } \Omega, \\
u(x, 0)=\psi(x) \text { on } \bar{D}, u(0, t)=u(1, t)=0 \text { for } 0<t \leq T,
\end{array}\right\}
$$

where $\delta(x)$ is the Dirac delta function, and $f$ and $\psi$ are given functions. This model is motivated by applications in which the ignition of a combustible medium is accomplished through the use of either a heated wire or a pair of small electrodes to supply a large amount of energy to a very confined area. When $q=1$, the model may also be used to describe the temperature $u$ of the channel flow of a fluid with temperature-dependent

Received April 26, 2007.

2000 Mathematics Subject Classification. Primary 35K60, 35K65, 35K57.

Key words and phrases. Degenerate semilinear parabolic first initial-boundary value problem, concentrated nonlinear source, critical position, global existence, blow-up.

E-mail address: chan@louisiana.edu

E-mail address: rxb1828@louisiana.edu 
viscosity in the boundary layer (cf. Chan and Kong [2]) with a concentrated nonlinear source at $b$; here, $x$ and $t$ denote the coordinates perpendicular and parallel to the channel wall respectively. When $q=0$, it can be used to describe the temperature of a onedimensional strip of a finite width that contains a concentrated nonlinear source at $b$. The case when $q=0$ was studied by Olmstead and Roberts [4] by analyzing its corresponding nonlinear Volterra equation of the second kind at the site of the concentrated source. A problem due to a source with local and nonlocal features was also studied by Olmstead and Roberts [5] by analyzing a pair of coupled nonlinear Volterra equations with different kernels. We assume that $f(0) \geq 0, f(u)$ and its derivatives $f^{\prime}(u)$ and $f^{\prime \prime}(u)$ are positive for $u>0$, and $\psi(x)$ is nontrivial, nonnegative and continuous such that $\psi$ attains its maximum at $b, \psi(0)=\psi(1)=0$, and

$$
\psi^{\prime \prime}+a \delta(x-b) f(\psi) \geq 0 \text { in } D .
$$

Chan and Tian [3] proved that under certain conditions, the solution $u$ of the problem (1.1) blows up in a finite time. For $q=0$, they and Olmstead and Roberts [4] showed that if the position $b$ of the concentrated source is sufficiently close to $x=0$ or $x=1$, then $u$ never blows up. The main purpose here is to find the exact position $b^{*}$ for the problem (1.1) with $q \geq 0$ such that $u$ never blows up for $b \in\left(0, b^{*}\right] \cup\left[1-b^{*}, 1\right)$, and $u$ always blows up in a finite time for $b \in\left(b^{*}, 1-b^{*}\right)$. This also implies that $u$ does not blow up in infinite time.

2. Critical position $b^{*}$. A proof similar to that used for Theorem 4 of Chan and Jiang [1] gives the following result.

ThEOREM 2.1. If $\lim _{t \rightarrow \infty} u(x, t)<\infty$, then $u(x, t)$ converges uniformly on $\bar{D}$ from below to a solution

$$
U(x)=a g(x ; b) f(U(b))
$$

of the nonlinear two-point boundary value problem

$$
-U^{\prime \prime}=a \delta(x-b) f(U(x)) \text { in } D, U(0)=U(1)=0,
$$

where

$$
g(x ; \xi)=\left\{\begin{array}{l}
\xi(1-x) \text { for } 0 \leq \xi \leq x \\
x(1-\xi) \text { for } x<\xi \leq 1
\end{array}\right.
$$

is Green's function corresponding to the problem (2.2).

When $\psi$ is sufficiently large and $f$ is sufficiently nonlinear, it follows from Theorem 3.3 of Chan and Tian [3] that there is a position $b$ to place the nonlinear concentrated source such that (1.1) blows up in a finite time. To find a position $b$ so that the solution $u$ exists for all $t>0$, let us first consider the problem (1.1) with $q=0$, namely,

$$
\left.\begin{array}{c}
\mu_{t}-\mu_{x x}=a \delta(x-b) f(\mu(x, t)) \text { in } \Omega \\
\mu(x, 0)=\psi(x) \text { on } \bar{D}, \mu(0, t)=\mu(1, t)=0 \text { for } 0<t \leq T .
\end{array}\right\}
$$


From Theorem 3.2 of Chan and Tian [3], the blow-up set is the single point $x=b$. From Chan and Tian [3],

$$
\mu(b, t)=a \int_{0}^{t} G(b, t ; b, \tau) f(\mu(b, \tau)) d \tau+\int_{D} G(b, t, \xi, 0) \psi(\xi) d \xi
$$

where

$$
G(x, t ; \xi, \tau)=2 \sum_{n=1}^{\infty}(\sin n \pi x)(\sin n \pi \xi) e^{-n^{2} \pi^{2}(t-\tau)} \text { for } t>\tau .
$$

From Olmstead and Roberts [4],

$$
\int_{0}^{t} G(b, t ; b, \tau) d \tau=b(1-b)-\frac{2}{\pi^{2}} \sum_{n=1}^{\infty} \frac{\sin ^{2} n \pi b}{n^{2}} e^{-n^{2} \pi^{2} t} .
$$

Since $\sum_{n=1}^{\infty}\left(\sin ^{2} n \pi b\right) e^{-n^{2} \pi^{2} t} / n^{2}$ and $2 \sum_{n=1}^{\infty}\left(\sin ^{2} n \pi b\right) e^{-n^{2} \pi^{2} t}$ converge uniformly in $(0, t)$, we have

$$
\begin{gathered}
\frac{\partial}{\partial t}\left(\int_{0}^{t} G(b, t ; b, \tau) d \tau\right)=2 \sum_{n=1}^{\infty}\left(\sin ^{2} n \pi b\right) e^{-n^{2} \pi^{2} t}>0 \\
\lim _{t \rightarrow \infty} \int_{0}^{t} G(b, t ; b, \tau) d \tau=b(1-b)-\frac{2}{\pi^{2}} \sum_{n=1}^{\infty} \frac{\sin ^{2} n \pi b}{n^{2}} \lim _{t \rightarrow \infty} e^{-n^{2} \pi^{2} t}=b(1-b) .
\end{gathered}
$$

The solution $v$ of the linear problem,

$$
v_{t}-v_{x x}=0 \text { in } \Omega, v(x, 0)=\psi(x) \text { on } \bar{D}, v(0, t)=v(1, t)=0 \text { for } 0<t \leq T,
$$

is given by

$$
v(x, t)=\int_{D} G(x, t ; \xi, 0) \psi(\xi) d \xi
$$

Since $\psi$ attains its maximum at $b$, it follows from the weak maximum principle that $v$ attains its maximum, denoted by $k_{0}$, at $(b, 0)$. From Theorem 2.4 of Chan and Tian [3], $\mu$ is a nondecreasing function of $t$. Hence, it follows from Theorem 2.6 of Chan and Tian [3] that for $0 \leq t \leq \theta, \mu(x, t)$ attains its maximum at $(b, \theta)$. Thus given any number $M>k_{0}$, it follows from (2.4) and (2.5) that

$$
\mu(b, t) \leq k_{0}+a f(M) \int_{0}^{t} G(b, t ; b, \tau) d \tau \leq k_{0}+a f(M) b(1-b) .
$$

In order that $k_{0}+a f(M) b(1-b) \leq M$ so that $\mu$ exists for all $t>0$, we choose $b$ in such a way that

$$
0<b \leq \frac{1}{2}\left[1-\sqrt{1-\frac{4\left(M-k_{0}\right)}{a f(M)}}\right] \text { or } \frac{1}{2}\left[1+\sqrt{1-\frac{4\left(M-k_{0}\right)}{a f(M)}}\right] \leq b<1 .
$$

Since $\mu$ is a nondecreasing function of $t$, we have for $0 \leq x \leq 1$ and $q>0$,

$$
x^{q} \mu_{t}-\mu_{x x} \leq \mu_{t}-\mu_{x x}
$$

which implies that the solution of the problem (1.1) is a lower solution of the problem (2.3). Thus under the above condition (2.6) on $b$, the solution of (1.1) also exists globally.

Let us consider a positive function $S(b)$ satisfying

$$
S(b)=a g(b ; b) f(S(b))=a b(1-b) f(S(b)) .
$$


Since $f(s)$ and its derivative $f^{\prime}(s)$ are positive for $s>0$, it follows that $f(S(b))$ and its derivative $f^{\prime}(S(b))$ are positive. We would like to know for $a$ sufficiently large, how $S(b)$ behaves as $b$ varies.

TheOREM 2.2. If $a>4 \sup _{S(b) \in(0, \infty)}\left(1 / f^{\prime}(S(b))\right)$, then

$S$ is a strictly increasing function of $b$ for $0<b<\frac{1}{2}\left(1-\sqrt{1-\frac{4}{a f^{\prime}(S(b))}}\right)$,

$S$ is a strictly decreasing function of $b$ for $\frac{1}{2}\left(1+\sqrt{1-\frac{4}{a f^{\prime}(S(b))}}\right)<b<1$.

Proof. A direct calculation gives

$$
S^{\prime}(b)=\frac{a(1-2 b) f(S(b))}{1-a b(1-b) f^{\prime}(S(b))} .
$$

For $b \in\left(0,\left(1-\sqrt{1-4 /\left(a f^{\prime}(S(b))\right)}\right) / 2\right)$, it follows from $a>4 \sup _{S(b) \in(0, \infty)}\left(1 / f^{\prime}(S(b))\right)$ that $b<1 / 2$, and hence $1-2 b>0$. Also,

$$
b<\frac{1}{2}\left(1-\sqrt{1-\frac{4}{a f^{\prime}(S(b))}}\right)
$$

gives

$$
b-\frac{1}{2}<-\frac{1}{2} \sqrt{1-\frac{4}{a f^{\prime}(S(b))}}<0 .
$$

Hence,

$$
\left(b-\frac{1}{2}\right)^{2}>\frac{1}{4}-\frac{1}{a f^{\prime}(S(b))}
$$

which gives

$$
1-a b(1-b) f^{\prime}(S(b))>0 .
$$

Thus, we have $S^{\prime}(b)>0$, and $S(b)$ is a strictly increasing function of $b$.

For $b \in\left(\left(1+\sqrt{1-4 /\left(a f^{\prime}(S(b))\right)}\right) / 2,1\right)$, we have

$$
\frac{1}{2}<\frac{1}{2}+\frac{1}{2} \sqrt{1-\frac{4}{a f^{\prime}(S(b))}}<b,
$$

and hence,

$$
\begin{gathered}
1-2 b<0, \\
b-\frac{1}{2}>\frac{1}{2} \sqrt{1-\frac{4}{a f^{\prime}(S(b))}}>0 .
\end{gathered}
$$

An argument as before gives

$$
1-a b(1-b) f^{\prime}(S(b))>0 .
$$

Thus, $S^{\prime}(b)<0$, and $S(b)$ is a strictly decreasing function of $b$. 
If $a>4 \sup _{U(b) \in(0, \infty)}\left(1 / f^{\prime}(U(b))\right)$, then it follows from (2.1) and Theorem 2.2 that on the interval $(0,1 / 2)$, the position $b$ for global existence of $u$ is closer to 0 than the position $b$ for the blow-up of $u$ in a finite time. On the other hand, on the interval $(1 / 2,1)$, the position $b$ for $u$ to exist globally is closer to 1 than the position $b$ for $u$ to blow up in a finite time. Thus, there exists $b^{*} \in(0,1 / 2)$ such that the steady state $U(x)$ exists for $b \in\left(0, b^{*}\right) \cup\left(1-b^{*}, 1\right)$, and does not exist for $b \in\left(b^{*}, 1-b^{*}\right)$. Since $u(x, t) \leq U(x)=$ $\lim _{t \rightarrow \infty} u(x, t)$ in $D \times(0, \infty)$ when $U$ exists, we have for $b \in\left(0, b^{*}\right) \cup\left(1-b^{*}, 1\right), u$ exists for $0 \leq t<\infty$, and for $b \in\left(b^{*}, 1-b^{*}\right), u$ always blows up in a finite time.

To calculate $b^{*}$, let us consider the steady state $U(x)$ of the problem (1.1). Since $u(x, t)$ attains its maximum at $(b, t)$, it follows that $U(x)$ attains its maximum at $x=b$. Also, $U(b)$ and $f(U(b))$ are positive. From (2.1),

$$
U(b)=a g(b ; b) f(U(b))=a b(1-b) f(U(b)) .
$$

Since $a f(U(b)) \neq 0$, we have

$$
b^{2}-b+\frac{U(b)}{a f(U(b))}=0
$$

which gives

$$
b=\frac{1}{2}\left(1 \pm \sqrt{1-\frac{4 U(b)}{a f(U(b))}}\right) .
$$

Since $b$ is real, we have

$$
a>4 \sup _{U(b) \in(0, \infty)} \frac{U(b)}{f(U(b))} .
$$

For the case $b=(1-\sqrt{1-4 U(b) /(a f(U(b)))}) / 2, b^{*}$ is the supremum of all $b$ such that $U(x)$ exists. Thus,

$$
b^{*}=\frac{1}{2}\left(1-\sqrt{1-\frac{4}{a} \sup _{U(b) \in(0, \infty)} \frac{U(b)}{f(U(b))}}\right) .
$$

Let us consider the function $g(s)=s / f(s)$. Then, $g^{\prime}(s)=0$ if and only if $s=$ $f(s) / f^{\prime}(s)$. Since $f$ is superlinear for $u$ to blow up in a finite time (cf. Theorem 3.3 of Chan and Tian [3]), we have $\lim _{s \rightarrow \infty} s / f(s)=0$. If we impose $f(0)>0$, then $\lim _{s \rightarrow 0^{+}} s / f(s)=0$. By Rolle's Theorem, $\sup _{U(b) \in(0, \infty)}(U(b) / f(U(b)))$ occurs when

$$
f(U(b))=U(b) f^{\prime}(U(b))
$$

where $0<U(b)<\infty$. This implies that $U(b)$ exists at $b=b^{*}$, and hence, $u$ does not blow up in infinite time.

In the case $b=(1+\sqrt{1-4 U(b) /(a f(U(b)))}) / 2,1-b^{*}$ is the infimum of all $b$ for which $U(x)$ exists. Thus,

$$
1-b^{*}=\frac{1}{2}\left(1+\sqrt{1+\frac{4}{a} \inf _{U(b) \in(0, \infty)} \frac{-U(b)}{f(U(b))}}\right) .
$$

An argument as above gives the conclusion that if $f(0)>0$, then $\inf _{U(b) \in(0, \infty)}(-U(b) / f(U(b)))$ occurs when $(2.8)$ holds with $0<U(b)<\infty$. Again, this implies that $U(b)$ exists at $b=1-b^{*}$, and hence, $u$ does not blow up in infinite time. 
We note that if (2.8) holds, then $U(b)$ can be determined. The above discussion gives the following result.

Theorem 2.3. If $a>4 \max \left\{\sup _{U(b) \in(0, \infty)}\left(1 / f^{\prime}(U(b))\right), \sup _{U(b) \in(0, \infty)}(U(b) / f(U(b)))\right\}$, then there exists $b^{*}$ given by $(2.7)$ such that for $b \in\left(0, b^{*}\right) \cup\left(1-b^{*}, 1\right), u$ exists for $0 \leq t<\infty$, and for $b \in\left(b^{*}, 1-b^{*}\right), u$ always blows up in a finite time. If in addition, $f(0)>0$, then $\sup _{U(b) \in(0, \infty)}(U(b) / f(U(b)))$ occurs when $(2.8)$ holds with $U(b) \in(0, \infty)$, and $u$ does not blow up in infinite time.

3. Examples. For illustration, we give below two examples on calculating $b^{*}$ for some given functions $f$.

EXAmple 3.1. Let $f(u)=(1+u)^{p}$, where $p$ is a real number greater than 1 . Since $f(0)>0$, it follows from $(2.8)$ that $U(b)=(1+U(b)) / p$, which gives

$$
U(b)=\frac{1}{p-1} .
$$

From Theorem 2.3 , if $a>4(p-1)^{p-1} / p^{p}$, then

$$
b^{*}=\frac{1}{2}\left[1-\sqrt{1-\frac{4(p-1)^{p-1}}{a p^{p}}}\right] .
$$

Thus for $b \in\left(0, b^{*}\right] \cup\left[1-b^{*}, 1\right), u$ exists for $0 \leq t<\infty$, and for $b \in\left(b^{*}, 1-b^{*}\right), u$ always blows up in a finite time.

EXAmPle 3.2. Let $f(u)=k e^{u}$, where $k$ is a positive number. Since $f(0)>0$, it follows from $(2.8)$ that $U(b)=1$. From Theorem 2.3, if $a>4 /(k e)$, then

$$
b^{*}=\frac{1}{2}\left(1-\sqrt{1-\frac{4}{a k e}}\right) .
$$

Thus for $b \in\left(0, b^{*}\right] \cup\left[1-b^{*}, 1\right), u$ exists for $0 \leq t<\infty$, and for $b \in\left(b^{*}, 1-b^{*}\right), u$ always blows up in a finite time.

A phenomenon related to blow-up is quenching. Chan and Jiang [1] studied the quenching phenomenon for the following semilinear problem with a concentrated nonlinear source at $b$,

$$
\begin{gathered}
L w=a \delta(x-b) f(w(x, t)) \text { in } \Omega, \\
w(x, 0)=0 \text { on } \bar{D}, w(0, t)=w(1, t)=0 \text { for } 0<t \leq T,
\end{gathered}
$$

where $\lim _{w \rightarrow c^{-}} f(w)=\infty$ for some positive constant $c$, and $f(w)$ and $f^{\prime}(w)$ are positive for $0 \leq w<c$. They showed that there exists a critical length $a^{*}$ such that for $a \leq a^{*}$, $w$ exists for $0 \leq t<\infty$, and for $a>a^{*}, \max \{w(x, t): 0 \leq x \leq 1\}$ reaches $c^{-}$, namely quenching occurs, in a finite time. Thus, existence of a critical domain of length $a^{*}$ in quenching is equivalent to existence of $b^{*}$ in blow-up in the sense that for $b \in$ $\left(0, b^{*}\right] \cup\left[1-b^{*}, 1\right)$, the solution exists for $0 \leq t<\infty$, and for $b \in\left(b^{*}, 1-b^{*}\right)$, the solution always blows up in a finite time. 


\section{References}

[1] C. Y. Chan and X. O. Jiang, Quenching for a degenerate parabolic problem due to a concentrated nonlinear source, Quart. Appl. Math. 62 (2004), 553-568. MR2086046 (2005e:35139)

[2] C. Y. Chan and P. C. Kong, Channel flow of a viscous fluid in the boundary layer, Quart. Appl. Math. 55 (1997), 51-56. MR1433751 (98c:35135)

[3] C. Y. Chan and H. Y. Tian, Single-point blow-up for a degenerate parabolic problem due to a concentrated nonlinear source, Quart. Appl. Math. 61 (2003), 363-385. MR1976376 (2004c:35173)

[4] W. E. Olmstead and C. A. Roberts, Explosion in a diffusive strip due to a concentrated nonlinear source, Methods Appl. Anal. 1 (1994), 435-445. MR1317023 (95k:35117)

[5] W. E. Olmstead and C. A. Roberts, Explosion in a diffusive strip due to a source with local and nonlocal features, Methods Appl. Anal. 3 (1996), 345-357. MR1421475 (97f:35110) 